\title{
Correspondence between retinotopic cortical mapping and conventional functional and morphological assessment of retinal disease
}

\author{
Markus Ritter, ${ }_{1}^{1}$ Allan Hummer, ${ }^{2}$ Anna A Ledolter, ${ }^{1}$ Graham E Holder, ${ }^{3,4,5}$ \\ Christian Windischberger, ${ }^{2}$ Ursula M Schmidt-Erfurth ${ }^{1}$
}

\begin{abstract}
- Additional material is published online only. To view please visit the journal online (http://dx.doi.org/10.1136/ 10.1136/bjophthalmol-2017311443)
\end{abstract}

${ }^{1}$ Department of Ophthalmology, Medical University of Vienna, Vienna, Austria

${ }^{2} \mathrm{MR}$ Center of Excellence, Center for Medical Physics and Biomedical Engineering, Medical University of Vienna, Vienna, Austria

${ }^{3}$ Department of Ophthalmology, National University of Singapore, National University Hospital, Singapore

${ }^{4} U C L$ Institute of Ophthalmology, University College London, London, UK ${ }^{5}$ Moorfields Eye Hospital, London, UK

Correspondence to Professor Ursula M SchmidtErfurth, Medical University of Vienna, Vienna 1090, Austria; ursula.schmidt-erfurth@ meduniwien.ac.at

Received 9 October 2017 Accepted 12 April 2018 Published Online First 26 April 2018

\begin{abstract}
Purpose The present study describes retinotopic mapping of the primary visual cortex using functional MRI (fMRI) in patients with retinal disease. It addresses the relationship between $\mathrm{FMRI}$ data and data obtained by conventional assessment including microperimetry (MP) and structural imaging.
\end{abstract}

Methods Initial testing involved eight patients with central retinal disease (Stargardt disease, STGD) and eight with peripheral retinal disease (retinitis pigmentosa, $R P$ ), who were examined using fMRI and MP (Nidek MP-1). All had a secure clinical diagnosis supported by electrophysiological data. fMRI used population-receptive field (pRF) mapping to provide retinotopic data that were then compared with the results of MP, optical coherence tomography and fundus autofluorescence imaging.

Results Full analysis, following assessment of fMRI data reliability criteria, was performed in five patients with STGD and seven patients with RP; unstable fixation was responsible for unreliable pRF measurements in three patients excluded from final analysis. The macular regions in patients with STGD with central visual field defects and outer retinal atrophy (ORA) at the macula correlated well with pRF coverage maps showing reduced density of activated voxels at the occipital pole. Patients with RP exhibited peripheral ORA and concentric visual field defects both on MP and pRF mapping. Anterior V1 voxels, corresponding to peripheral regions, showed no significant activation. Correspondence between MP and pRF mapping was quantified by calculating the simple matching coefficient.

Conclusion Retinotopic maps acquired by fMRI provide a valuable adjunct in the assessment of retinal dysfunction. The addition of microperimetric data to $\mathrm{pRF}$ maps allowed better assessment of macular function than MP alone. Unlike MP, pRF mapping provides objective data independent of psychophysical perception from the patient.

\section{INTRODUCTION}

There are many methods of examining function in patients with retinal disease. Some, such as perimetry or visual acuity testing, are psychophysical, while others, such as electrophysiology, provide an objective assessment of function. Microperimetry (MP) is a method of localised testing of retinal sensitivity in the foveal, parafoveal and peripheral macular regions. ${ }^{1}$ It allows structure-function correlation and is increasingly used in clinical trials investigating new therapies. However, as a psychophysical test, MP results critically depend on patient performance and active feedback. Learning effects are well recognised, ${ }^{2}$ and there may also be ceiling and floor effects in MP devices with limited luminance range. ${ }^{3}$ Such issues limit the power of MP as a diagnostic technique and its applicability as an outcome measure to document therapeutic efficacy in slowly progressive macular diseases.

Retinotopic mapping using functional MRI (fMRI) represents an alternative method based on MRIs acquired with blood oxygenation level-dependent (BOLD) contrast to reveal areas of neuronal activity in the visual cortex. ${ }^{4}$ In particular, population-receptive field ( $\mathrm{pRF}$ ) mapping, as an extended retinotopic mapping technique, has the potential for visual field mapping independent of patient performance. ${ }^{5}$ In $\mathrm{pRF}$ mapping, the term 'receptive field' establishes a connection between a visually stimulated retinal region and an area in the visual cortex that responds to this stimulation with an increased BOLD signal.

Several studies have investigated the cortical responses to visual stimuli in the context of retinal lesions. The first human fMRI data in this context were obtained from a patient with age-related macular degeneration (AMD) and bilateral macular lesions, where there was a large unresponsive zone in V1 corresponding to the lesion.? Subsequent investigations confirmed silent cortical zones matching patients' visual field defects. ${ }^{7-11}$ Others reported reorganisation of visual processing in persons with retinal dysfunction secondary to different retinal diseases. ${ }^{12-15}$ However, the extent and mechanisms of such reorganisation remain controversial.

The purpose of the present study is to compare the results of $\mathrm{pRF}$ mapping, MP and conventional structural imaging. Apart from healthy controls, two illustrative patient groups were selected to cover distinct patterns of lesion subtypes. Stargardt disease (STGD; ABCA4 retinopathy), the most common juvenile macular dystrophy, often has sharply demarcated central macular atrophy and central visual field loss. In contrast, patients with retinitis pigmentosa (RP) have constricted visual fields but can have well-preserved central visual function, particularly early in the disease. The study also introduces a novel approach for linking retinal function assessed with MP to cortical pRF data. 
Table 1 Patient data, fMRI data reliability and microperimetry fixation stability

\begin{tabular}{|c|c|c|c|c|c|c|c|c|c|c|c|c|}
\hline $\begin{array}{l}\text { Disease/Patient } \\
\text { number }\end{array}$ & Sex & Age & $\begin{array}{l}\text { Measured } \\
\text { eye }\end{array}$ & $\begin{array}{l}\text { VA } \\
\text { logMAR }\end{array}$ & $\begin{array}{l}\text { Full-field ERGs } \\
\text { scotopic/photopic }\end{array}$ & $\begin{array}{l}\text { Eccentric } \\
\text { fixation }\end{array}$ & $\begin{array}{l}\% \text { MP1 } \\
\text { fixation } \\
2^{\circ}\end{array}$ & $\begin{array}{l}\% \mathrm{MP} 1 \\
\text { fixation } 4^{\circ}\end{array}$ & $\begin{array}{l}\text { MP } \\
\text { fixation } \\
\text { stability }\end{array}$ & $\begin{array}{l}\text { Number } \\
\text { of } p R F \\
\text { mapping } \\
\text { runs }\end{array}$ & $\begin{array}{l}\text { pRF } \\
\text { stability } \\
(\mathrm{MAE})\left(^{\circ}\right)\end{array}$ & $\begin{array}{l}\text { Framewise } \\
\text { displacement } \\
(\mathrm{mm})\end{array}$ \\
\hline STGD 1 & $\mathrm{~F}$ & 20 & OD & 0.1 & $\mathrm{n} / \mathrm{n}$ & No & 99 & 100 & Stable & 3 & 0.91 & $0.17 \pm 0.03$ \\
\hline STGD 2 & $\mathrm{~F}$ & 35 & OD & 0.9 & $n / n$ & Yes & 62 & 95 & $\begin{array}{l}\text { Relatively } \\
\text { unstable }\end{array}$ & 3 & 2.56 & $0.35 \pm 0.03$ \\
\hline STGD 3 & $\mathrm{~F}$ & 50 & $O D$ & 0 & $n / n$ & No & 100 & 100 & Stable & 4 & 0.75 & $0.13 \pm 0.01$ \\
\hline STGD 4 & $\mathrm{~F}$ & 73 & $O D$ & 0.9 & $n / n$ & Yes & 11 & 42 & Unstable & 2 & 0.51 & $0.64 \pm 0.23$ \\
\hline STGD 5 & $\mathrm{~F}$ & 33 & OD & 0.9 & $n / n$ & Yes & 20 & 70 & Unstable & 4 & 1.62 & $0.18 \pm 0.01$ \\
\hline STGD 6 & $\mathrm{~F}$ & 18 & OS & 0.5 & $n / n$ & No & 93 & 99 & Stable & 4 & 0.96 & $0.16 \pm 0.01$ \\
\hline STGD 7 & $\mathrm{~F}$ & 27 & OS & 0.7 & $n / n$ & No & 67 & 98 & $\begin{array}{l}\text { Relatively } \\
\text { unstable }\end{array}$ & 4 & 0.62 & $0.17 \pm 0.02$ \\
\hline STGD 8 & $\mathrm{M}$ & 21 & $O D$ & 0.9 & $n / n$ & Yes & 45 & 84 & $\begin{array}{l}\text { Relatively } \\
\text { unstable }\end{array}$ & 4 & 0.96 & $0.26 \pm 0.03$ \\
\hline RP 1 & M & 46 & $O D$ & 0.1 & $u / u$ & No & 99 & 100 & Stable & 4 & 0.62 & $0.28 \pm 0.05$ \\
\hline RP 2 & $\mathrm{~F}$ & 47 & OD & 0 & $\mathrm{u} / \mathrm{u}$ & No & 98 & 100 & Stable & 4 & 0.67 & $0.22 \pm 0.03$ \\
\hline RP 3 & M & 51 & OS & 0.1 & $u / u$ & No & 95 & 99 & Stable & 2 & 1 & $0.27 \pm 0.04$ \\
\hline RP 4 & $\mathrm{~F}$ & 42 & OS & 0 & $\mathrm{u} /$ severely reduced & No & 100 & 100 & Stable & 3 & 0.6 & $0.35 \pm 0.04$ \\
\hline RP 5 & $\mathrm{~F}$ & 36 & $O D$ & 0.1 & $u / u$ & No & 97 & 99 & Stable & 4 & 0.54 & $0.17 \pm 0.01$ \\
\hline RP 6 & M & 38 & OD & 0 & u/severely reduced & No & 98 & 100 & Stable & 4 & 0.77 & $0.17 \pm 0.04$ \\
\hline RP 7 & $\mathrm{~F}$ & 43 & OD & 0.1 & $u / u$ & No & 95 & 100 & Stable & 4 & 0.6 & $0.22 \pm 0.01$ \\
\hline RP 8 & $\mathrm{~F}$ & 31 & OD & 0.1 & $\mathrm{u} / \mathrm{u}$ & No & 94 & 95 & Stable & 4 & 0.79 & $0.27 \pm 0.01$ \\
\hline
\end{tabular}

Fixation was classified as stable when $75 \%$ of fixations were located within a $2^{\circ}$ circle, as relatively unstable when $75 \%$ of fixations were located within a $4^{\circ}$ circle and as unstable when less than $75 \%$ of fixations were located within a $4^{\circ}$ circle.

ERG, electroretinogram; $F$, female; $f M R I$, functional MRI; logMAR, logarithm of the minimum angle of resolution; $M$, male; MAE, mean average error; $M P$, microperimetry; $n$, normal; OD, right eye; OS, left eye; pRF, population-receptive field; RP, retinitis pigmentosa; STGD, Stargardt disease; VA, visual acuity; $u$, undetectable.

\section{PATIENTS AND METHODS}

Initially, eight patients with STGD (one male, seven female; age: $34.6 \pm 18.7$ years) and eight patients with RP (three male, five female; age: $41.8 \pm 6.5$ years) were examined. Inclusion criteria for patients with STGD were mutations in the $A B C A 4$ gene; a central, well-demarcated atrophic macular lesion; a central scotoma not exceeding $15^{\circ}$ visual angle diameter; and normal full-field electroretinograms (ERG) (ie, all patients were in group 1 according to the criteria of Lois $e a^{16}$ ). The patients with RP had a secure clinical diagnosis supported by imaging studies and electrophysiological data. Full-field ERGs, recorded in accordance with the International Society for Clinical Electrophysiology of Vision standards, ${ }^{17}$ showed undetectable or severely reduced scotopic (rod system-dominated) and photopic (cone system) responses consistent with generalised retinal dysfunction at the level of the photoreceptor. All patients with RP had preserved central macular function with best-corrected visual acuities (BCVA) of $\geq 20 / 25$ and visual fields constricted to $<10^{\circ}$ visual angle diameter (please refer to table 1 for details). In addition, two healthy subjects (both female; age 22 and 27) were recruited to show the performance of pRF mapping using simulated scotomata.

Written informed consent was obtained from all subjects before their participation.

\section{Clinical examination}

Patients underwent a full ophthalmic examination including slit lamp examination and dilated fundus examination. BCVA was measured using ETDRS charts.

\section{Imaging}

Blue-light fundus autofluorescence (FAF) and spectral domain optical coherence tomography (SD-OCT) images were recorded using a Spectralis HRA \& OCT system (Heidelberg Engineering, Heidelberg, Germany).

\section{Microperimetry}

Macular function was assessed by an MP-1 microperimeter (Nidek, Padova, Italy). The stimulus intensity ranged from 0 $\mathrm{dB}$ to $20 \mathrm{~dB}$ in $1 \mathrm{~dB}$ steps, with the initial intensity at $12 \mathrm{~dB}$. The stimulus was a grid pattern consisting of 41 stimuli $\left(12^{\circ} \times 12^{\circ}\right)$. Fixation stability was assessed as part of the microperimetric examination. $^{18}$

\section{fMRI measurements}

Functional images of the visual cortex were recorded on a 3T TIM Trio scanner (Siemens Medical, Erlangen, Germany) with the lower part of a 32-channel head coil (corresponding to a 20-channel head coil) and using the Center for Magnetic Resonance Research multiband-accelerated echo planar imaging sequence $^{19}$ (Echo Time (TE)/Repetition Time (TR)=36/1500 ms, multiband factor 2,28 coronal slices, voxel size $=1 \times 1 \times 1$ $\mathrm{mm}^{3}$ ). In addition, structural images were acquired using a magnetisation-prepared rapid gradient-echo sequence (TE/ $\mathrm{TR}=4.21 / 2300 \mathrm{~ms}, 160$ sagittal slices, voxel size $=1 \times 1 \times 1 \mathrm{~mm}^{3}$, field of view $=256 \mathrm{~mm}$ ).

\section{Stimuli}

The stimulus consisted of an isoluminant reversing checkerboard bar $\left(8 \mathrm{~Hz}\right.$, width $\left.2.35^{\circ}\right)$ moving across a mean-luminance screen (total width $18.8^{\circ}$ ) in eight different directions. The bar crossed the screen in 24 discrete steps, each separated by $0.78^{\circ}$ visual angle in space and TR=1.5 $\mathrm{s}$ in time, which amounted to the duration of $5 \mathrm{~min} 36 \mathrm{~s}$ for a single run. Patients were instructed to fixate on a small dot (12 pixels or $0.22^{\circ}$ visual angle diameter) 
at the centre of the screen. Thin diagonal lines crossing at the centre dot were also displayed to assist patients in maintaining stable fixation. Two to four runs were recorded per participant, which were concatenated to form a single data set for analysis.

\section{fMRI analysis}

Data sets underwent standard fMRI preprocessing consisting of slice timing correction, realignment and $2 \mathrm{~mm}$ full width at half maximum smoothing using SPM V.12 (www.fil.ion.ucl.ac. uk/spm/, Wellcome Trust Centre for Neuroimaging, London, UK). White and grey matter tissues were segmented using the FreeSurfer image analysis suite (http://surfer.nmr.mgh.harvard. edu/). After manual topographical correction, all grey matter voxels were submitted for analysis. Fitting of the pRF model was performed using a two-dimensional (2D) Gaussian approach ${ }^{5}$ on mrVista (Stanford University, Stanford, California). In short, the optimal model for each voxel was obtained by creating a set of time-series corresponding to the pRF stimulation patterns which were fitted to the BOLD fMRI data. The optimal 2D Gaussian of a voxel was then found by varying pRF position $(\mathrm{x}, \mathrm{y})$ and size $(\sigma)$ in a way that the residual sum of squares between measured and modelled fMRI time-course was minimised. All maps of pRF parameters were thresholded at 10\% explained variance.

\section{Assessment of fMRI data reliability \\ Framewise displacement}

The quality of fMRI data can be assessed in various ways. One of the most important parameters relates to patient movement during data acquisition and is referred to as framewise displacement (FD). This parameter is obtained during preprocessing where images at individual time instances are realigned to the mean (temporal average) image. The average values for each patient are given in table 1. Based on the FD threshold proposed by Power et al, ${ }^{20}$ patients exhibiting mean FD values above 0.5 $\mathrm{mm}$ were excluded from further analysis.

\section{Retinotopic mapping stability}

A powerful measure for retinotopic map reliability is stability across multiple runs. In this study, two to four pRF mapping runs were conducted for each patient, the number depending on the individual patient compliance (eg, fatigue). pRF stability of the eccentricity parameter was determined by calculating the mean average error (MAE) between all runs as described previously. ${ }^{21}$ Patients were excluded when exceeding an MAE of $1^{\circ}$ visual angle, correlating to about $5 \%$ of the stimulus extent. The MAE values of each patient are shown in table 1.

\section{RESULTS}

Three patients with STGD failed to meet the fMRI data reliability criteria due to $\mathrm{pRF}$ instability and/or movement during fMRI measurements. These patients also demonstrated relatively unstable or unstable fixation measurements on MP. One patient with RP was excluded since there were no active voxels, that is, no voxels explaining more than $10 \%$ variance, present in V1 after pRF analysis (see table 1). Five patients with STGD and seven patients with RP remained for full analysis.

\section{Correlation of MP, structural imaging and visual field coverage}

pRF mapping in healthy participants with simulated scotoma

Figure 1A,C shows the eccentricity map of healthy control participants overlaid on the white matter/grey matter surface mesh. These subjects were presented with an artificial central scotoma
Healthy Controls

Artificial Central Scotoma $\left(\varnothing=9.4^{\circ}\right)$

$1 \mathrm{~A}$
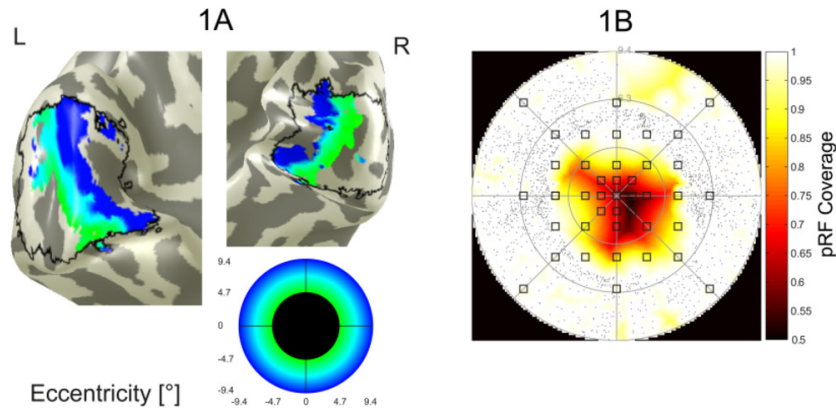

Artificial Quadrant Scotoma

$1 \mathrm{C}$

1D

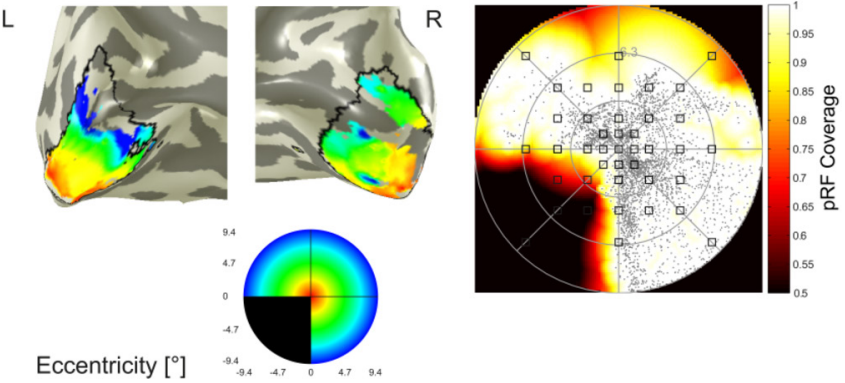

Figure 1 Results from two healthy subjects with an artificial central and an artificial quadrant scotoma. For full stimulation, that is, a regular run, $A$ and $C$ show the eccentricity maps overlaid on the cortex, while $B$ and $D$ show the microperimetry test grid overlaid on the pRF coverage maps calculated from the functional MRI data. Each dot represents the centre of a receptive field of a single voxel and every pRF centre is associated with a two-dimensional Gaussian, which together constitute the coverage map. pRF, population-receptive field.

(figure 1A) and an artificial scotoma in the lower left quadrant (figure 1C) for fMRI measurements. As expected, eccentricity increases towards the more anterior regions of the visual cortex. The polar angle map, thresholded at $1 \%$ explained variance, was used to delineate the primary visual cortex. It shows the horizontal meridian at the centre of $\mathrm{V} 1$ and the vertical meridian at the V1/V2 border. Analysis results show no significant activation voxels representing central retinal areas, corresponding to simulated scotomatous regions. Figure 1B,D depicts the MP test grid overlaid on pRF coverage maps obtained by plotting the pRF centre of every suprathreshold voxel along with its width (pRF size). The height of the combined Gaussians is then colour-coded, with black representing low pRF coverage and therefore a possible scotoma. Using this approach it is possible to detect and locate the artificial scotoma in the pRF coverage plot without modifying the analysis pipeline.

pRF mapping in patients with retinal scotoma

Similar to the healthy control in figure $1 \mathrm{~A}$, figure $2 \mathrm{~A}$ shows the eccentricity map of a patient with STGD. From the pRF results it can be clearly seen that this patient with STGD suffers from a central scotoma with foveal-sparing, as significant activation can be observed at the occipital pole corresponding to the fovea. Figure 2B depicts the MP results in more detail overlaid on the pRF coverage map and also demonstrates an annular scotoma with central sparing. An area of outer retinal 
STGD 1

$2 \mathrm{~A}$

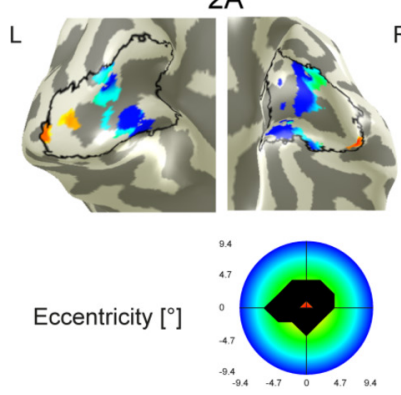

$2 \mathrm{C}$
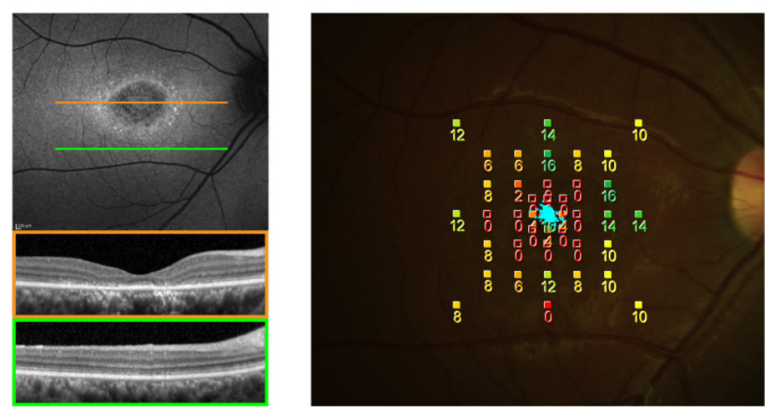

Figure 2 Results of the right eye of a patient with fovea-sparing STGD. The preserved central visual function, spanning about $1^{\circ}$ visual angle, is clearly visible in the eccentricity map (A) and coverage plot (B). On FAF imaging (C, top), a characteristic ring-shaped hypofluorescent area surrounded by small hyperfluorescent flecks is visible. It corresponds to parafoveal regions showing loss of photoreceptor layers/retinal pigment epithelium as shown on a central SD-OCT B-scan (C, bottom). Note the sparing of central foveal anatomy on both FAF and OCT corresponding to functional MRI activation at the top of the occipital pole. (D) The superimposition of the microperimetry on the fundus. The blue dots indicate the patient's fixation pattern. FAF, fundus autofluorescence; $\mathrm{PRF}$, population-receptive field; SD-OCT, spectral domain optical coherence tomography; STGD, Stargardt disease.

atrophy corresponding to the functional findings is present on FAF (figure 2C, top) and SD-OCT imaging (figure 2C, bottom, orange line). MP measurements suggest a possible additional small area of severely reduced macular sensitivity $(0 \mathrm{~dB})$ outside the ring scotoma in the inferior macular region, which is not visible on the pRF coverage map. As no corresponding changes are visible on either SD-OCT or FAF imaging (figure 2C, bottom, green line), which would be expected with such marked sensitivity loss, the pRF mapping corresponds better with retinal imaging than MP in this specific region.

Figure 3A shows a patient with STGD lacking foveal-sparing and with a complete central scotoma. In contrast to figure $2 \mathrm{~A}$, the function near the occipital pole is not preserved. Figure 3B shows a strong agreement of both modalities. Concordant with the functional data, a hypofluorescent area was present on FAF (figure 3C, top) and nearly complete atrophy of the central outer retinal layers on SD-OCT imaging (figure 3C, bottom).

Figure 4A depicts a patient with RP (genetic diagnosis unavailable). The patient has a concentric peripheral scotoma detectable both by MP and pRF mapping. Anterior V1 voxels, corresponding to peripheral regions, show no significant activation. Figure 4B shows similar results, with both methods also demonstrating moderately reduced central visual function. Loss of retinal pigment epithelium (RPE) and photoreceptor layers in
$3 A$

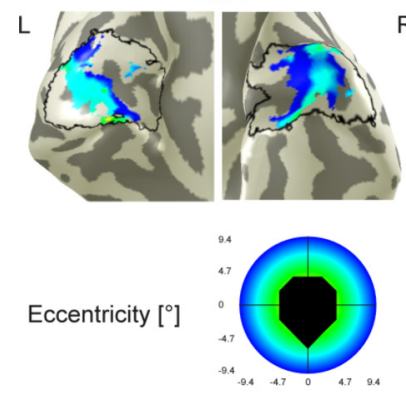

$3 \mathrm{C}$
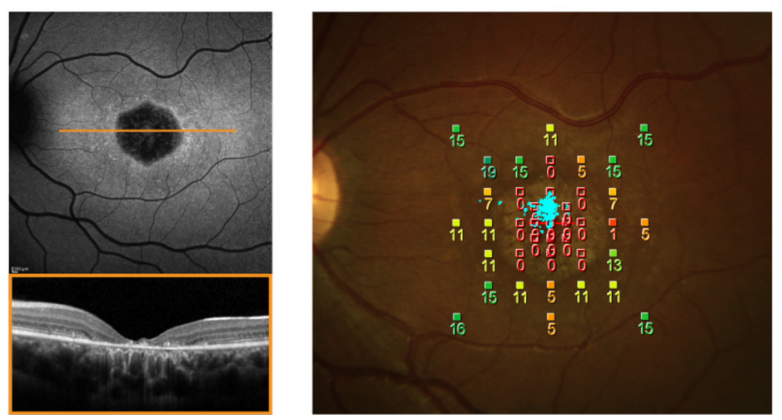

Figure 3 (A) Results of the left eye of a patient with more classical STGD. The microperimetry is roughly depicted by the eccentricity colour circle. It is shown in detail in B. FAF imaging shows central hypofluorescence with hyperfluorescent borders corresponding to loss of outer retinal structure on OCT (C). (D) Microperimetry superimposed on the fundus. The blue dots indicate the patient's fixation pattern. Note the lack of activation at the occipital pole in the fMRI corresponding to the area of loss of structure on FAF and OCT. Note also the similarity between the fMRI data in this clinical patient with the fMRI data in figure 1 obtained from a normal subject using an experimental scotoma of similar size. FAF, fundus autofluorescence; fMRI, functional MRI; OCT, optical coherence tomography; pRF, population-receptive field; STGD, Stargardt disease.

the corresponding peripheral macular regions is present on both FAF (figure 4C, top) and SD-OCT imaging (figure 4C, bottom).

\section{Quantitative comparison}

Coverage maps originating from MP and $\mathrm{pRF}$ mapping were compared on a subject-by-subject basis. Results were binarised, because in contrast to MP, pRF mapping only uses a single intensity stimulus and regions exhibiting low $\mathrm{pRF}$ coverage are therefore not equivalent to $\mathrm{MP}$ test points with low $\mathrm{dB}$ values. Areas of low pRF coverage are not necessarily more responsive to higher stimulus intensities. PRF coverage maps range from 0 to 1 and were thresholded at a value of 0.7 . Everything below this threshold was labelled as scotoma, while suprathreshold areas were labelled as functional. For MP every test point with a value higher than $0 \mathrm{~dB}$ was defined as functional. Binarised coverage maps, which show the correspondence between MP and pRF mapping, are depicted in figure 5 for patients with STGD and in figure 6 for patients with RP. To quantitatively compare the different modalities, $\mathrm{pRF}$ coverage values at the $41 \mathrm{MP}$ test point positions were sampled. Correspondence was calculated with the simple matching coefficient (SMC) defined by:

$$
S M C=\frac{\text { Number of identical test points }}{\text { Number of total test points }}
$$


RP 1

$4 \mathrm{~A}$

$4 \mathrm{C}$

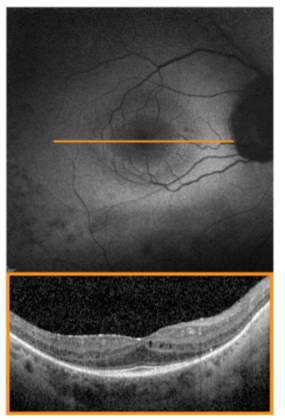

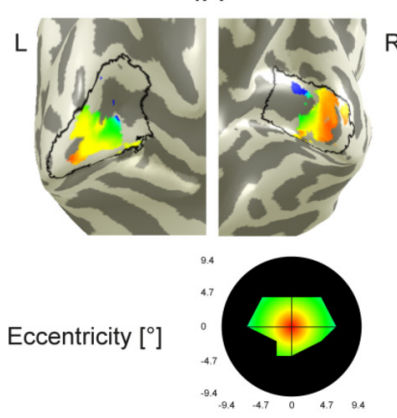

4B

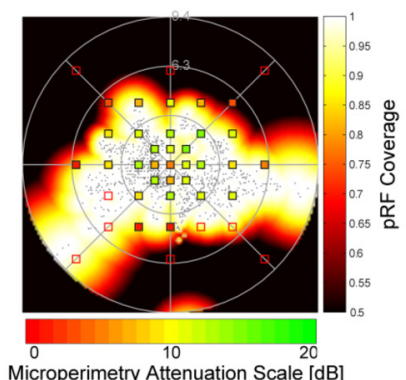

4D

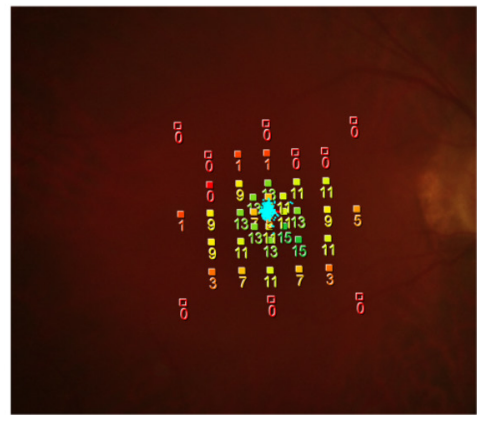

Figure 4 Results of the right eye of a patient with RP. (A) shows the eccentricity maps overlaid on the cortex, while (B) depicts the test grid overlaid on the PRF coverage mapscalculated from functional MRIdata. FAF imaging and OCT imaging (C) show marked loss of the outer retina in the peripheral macula and a few central intraretinal cysts. There is sparing of the structure of central retina. (D) Microperimetry superimposed on the fundus. The blue dots indicate the patient's fixation pattern. Note the well-preserved activation of the occipital pole delineating the extent of cortical activation and corresponding well to the retinal structural imaging data, with loss of the BOLD signal corresponding to peripheral macular regions. BOLD, blood oxygenation level-dependent; FAF, fundus autofluorescence; $\mathrm{OCT}$, optical coherence tomography; pRF, population-receptive field; $R P$, retinitis pigmentosa.

which ranges from 0 (all test point values differ) to 1 (the values of all test points are identical). For patients with STGD, three of five patients show an SMC higher than 0.8. STGD patient 1 showed lower agreement due to a peripheral scotoma reported in MP, which is discussed above. STGD patient 3 shows a high agreement, but no scotoma is evident in the pRF coverage. This could be due to limitations such as stimulus design and functional run repetitions, as discussed in Hummer et $a l,{ }^{22}$ where only artificial scotoma with a radius larger than $2.35^{\circ}$ visual angle could be reliably detected in single subjects using pRF mapping. STGD patient 7 shows a central scotoma in both the MP and pRF coverage maps. However, in the pRF coverage map, this scotoma extends to the upper visual field. A reason for this mismatch could be the instability of pRF parameters near the borders of different visual cortex areas, which correspond to the vertical meridian in the visual field map. The coverage map of the second healthy subject also hints at this instability. Six of seven patients with RP show an SMC higher than 0.8 . For $\mathrm{RP}$ patient 7, the coverage map is far more complete in the pRF approach. pRF sizes are large for voxels associated with peripheral regions, which means that relatively few voxels can lead to such an effect.
Online supplementary figures 1 and 2 show the underlying functional data for figures 5 and 6, namely the non-binarised MP and V1 pRF maps of the eccentricity parameter overlaid on the visual cortex mesh of each subject.

\section{DISCUSSION}

This study reports fMRI-based pRF retinotopy in the primary visual cortex of patients with STGD and RP. The clear differences in the clinical features between the two groups of patients, with the patients with STGD having central macular involvement with peripheral sparing and the patients with RP having peripheral involvement with central sparing, are accurately reflected in the fMRI data, which therefore complement MP and structural imaging. Although some previous studies have reported comparison in a variety of visual pathway disorders such as AMD, glaucoma, RP or optic radiation lesions, ${ }^{7} 152324$ this is the first to address comparisons between MP and pRF mapping in patients with localised retinal dysfunction, and further to compare those data with the results of structural retinal imaging.

Data in patients with STGD confirm previous studies reporting unresponsive zones in V1 in the central macular lesions and peripheral macular sparing. ${ }^{7-11}$ Other authors ${ }^{12-14}$ have shown activation of 'foveal' cortex by peripheral stimuli in patients with macular degeneration, which they attributed to cortical reorganisation. Fundamental to any future clinical utility of $\mathrm{fMRI}$ is that the imaging corresponds to the clinical presentation. Inspection of the data in figures $3 \mathrm{~A}$ and $1 \mathrm{~A}$, the former a patient with a central scotoma due to STGD and the latter from a healthy participant and an experimental scotoma of similar size to that of the patient with STGD, shows a strong similarity, demonstrating that there is potential for fMRI to play a significant clinical role in the future. The corresponding area of loss of central retinal structure is clearly visible both on FAF and OCT, again correlating with fMRI. Results in patients with RP are also consistent with previous reports, ${ }^{25} 26$ demonstrating occipital pole activation corresponding to the remaining central retinal structure as shown on FAF and OCT.

However, there is not $100 \%$ correspondence between the MP-derived retinal sensitivity maps and pRF mapping, and this may relate to differences in methodology. MP relies on a threshold detection profile, whereas fMRI uses a suprathreshold temporally modulated patterned stimulus of constant mean luminance. Further, MP uses a spot stimulus with a defined shape and size, whereas fMRI uses a bar moving across a screen while exposing a reversing checkerboard stimulus. A further possibility is that the mismatches may arise from subtle cortical reorganisation processes. As suggested previously, ${ }^{12-14}$ the occurrence of reorganisation might depend on the extent and duration of visual loss. These aspects could be important issues for further clarification in future research.

One potential use of fMRI could be in the evaluation of patients receiving therapeutic interventions such as gene or cell replacement therapy, where the possibility of cortical reorganisation could occur, and where restoration of function to an area of the retina that has previously lost function could be demonstrated objectively at a cortical level even if difficult to demonstrate objectively with electrophysiology at a retinal level. It has been shown that retinotopic eccentricity may be shifted in patients with RP such that central retinal inputs are represented in more peripheral locations in $\mathrm{V} 1 .{ }^{15}$ However, it has also been shown that task-dependent stimuli can result in BOLD responses being detectable in relation to a previously unresponsive 'lesion 

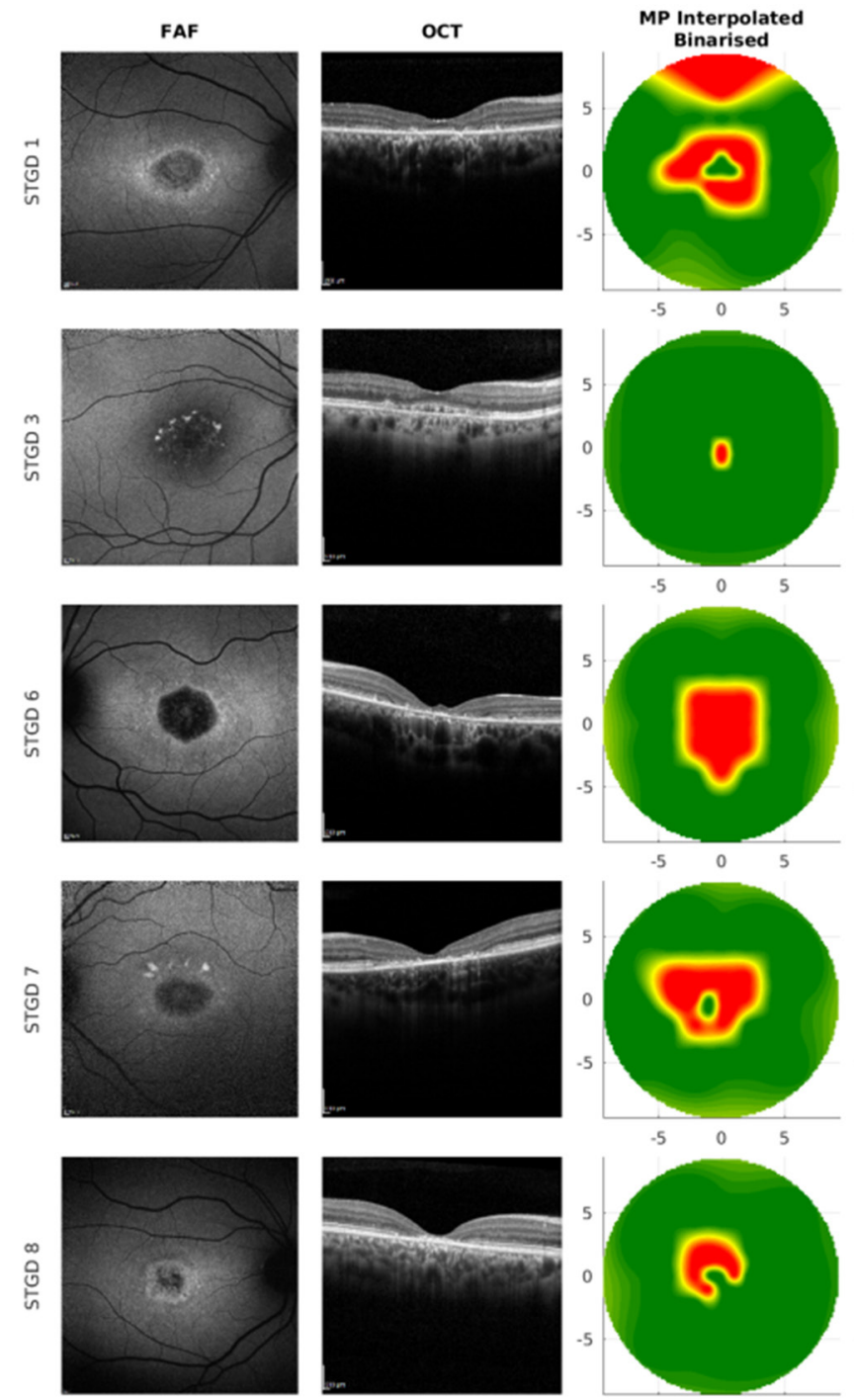

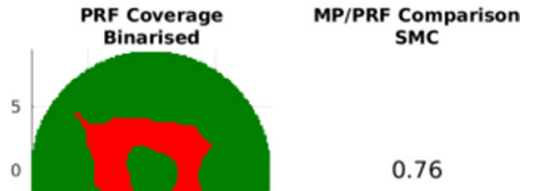

0.95

0.93

0.68

0.88
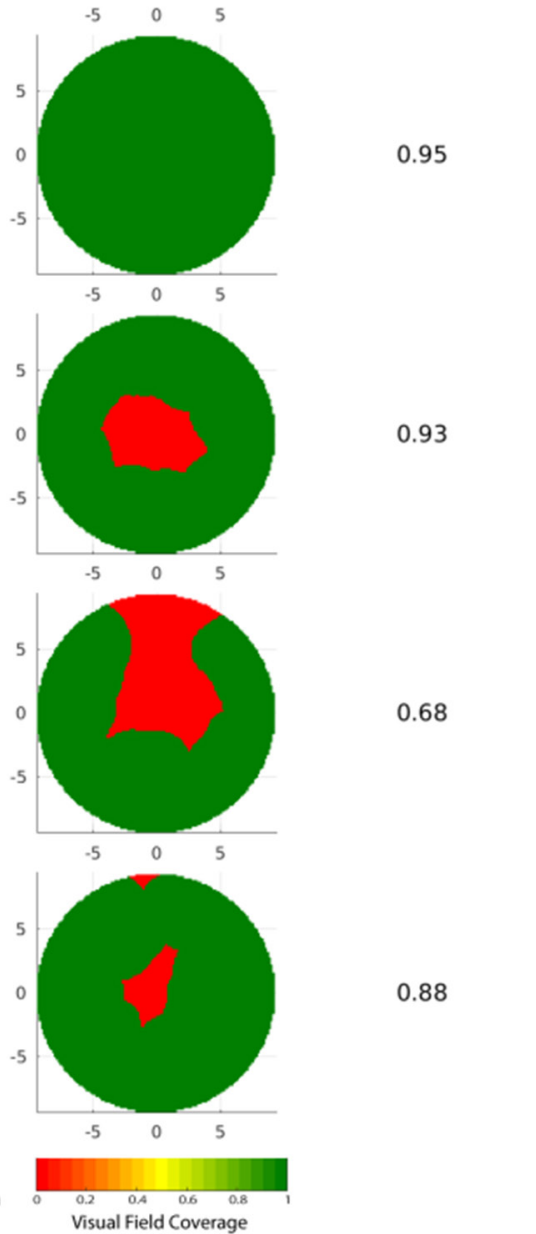

Visual Field Coverage

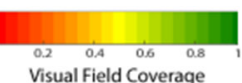

Figure 5 Comparison of data derived from FAF, OCT, MP and pRF mapping for five patients with STGD. The left column displays the FAF results while the second column SD-OCTs for every subject showing central macular areas of outer retinal atrophy corresponding to the functional findings. The third column shows MP data, which were first binarised and then interpolated. The fourth column shows the binarised pRF coverage of each subject. The fifth column shows the SMC of MP versus PRF mapping, which was calculated based on the 41 MP test points and pRF mapping data sampled from the pRF coverage map at these locations. FAF, fundus autofluorescence; MP, microperimetry; pRF, population-receptive field; SDOCT, spectral domain optical coherence tomography; SMC, simple matching coefficient.

projection zone', ${ }^{26}$ but which may not relate to any cortical reorganisation.

Some patients who were initially considered were excluded from the correlation of MP and visual field coverage when there were no active voxels (one RP) or due to pRF instability and/ or movement during fMRI measurements (three STGD), with all three patients with STGD demonstrating instability of fixation on MP, suggesting that fixation stability is the major limiting factor in fMRI measurements and greatly reduces the quality of $\mathrm{pRF}$ mapping results. The application of gaze correction based on eye-tracker data as part of the analysis pipeline may help. ${ }^{21}$ As unstable fixation is common early in STGD and in the more advanced stages of RP, future studies to evaluate the use of eye-tracker system in such patients are essential for any future clinical application and could reduce the high dropout rate. Since MP, currently the clinical investigation method of choice for measurement of localised retinal macular function, is subjective and unreliable for the differentiation of placebo and treatment effects in these slowly progressing diseases, the development of adequate clinical measures to assess functional restoration objectively is strongly needed. Strict inclusion criteria were employed in order to create an ideal setting to evaluate a possible correspondence between MP and pRF mapping. The low prevalence of patients fulfilling these criteria limits the number of patients available for a single-centre study. While the results may offer a contribution towards best practice methods in fMRI utilisation for monitoring macular dysfunction both in terms of documentation of the natural course of the disease and 

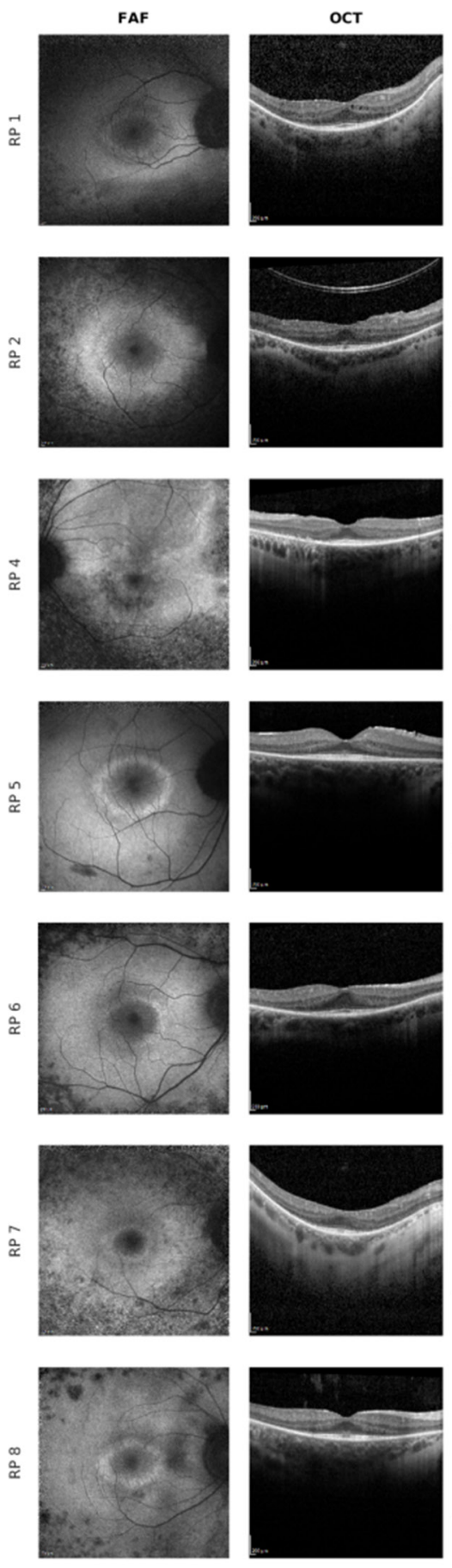
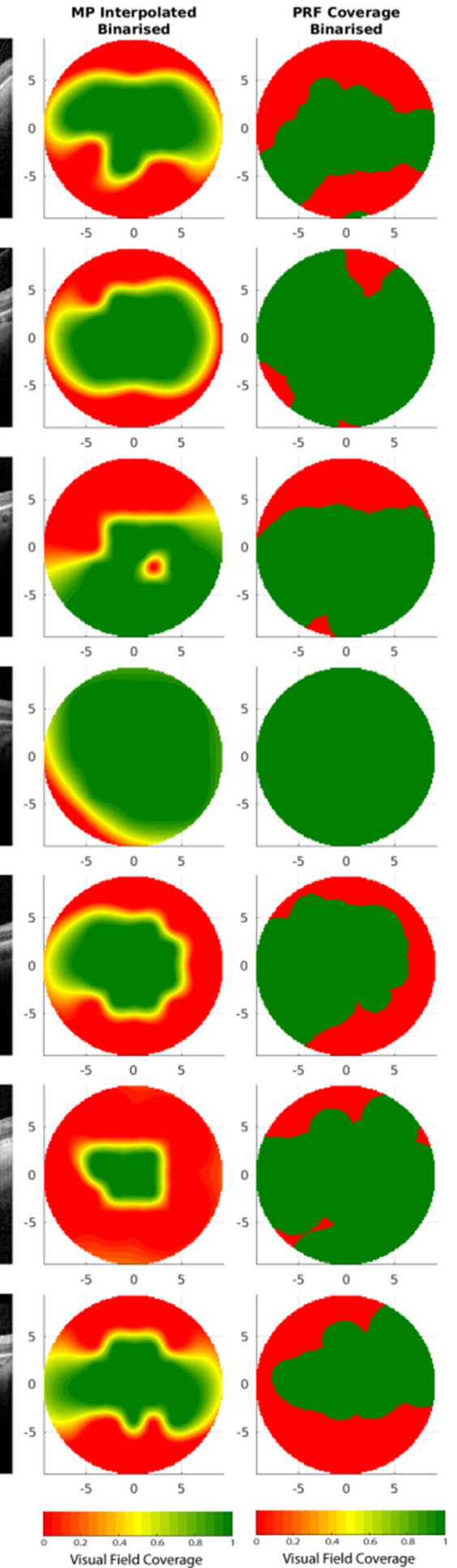

MP/PRF Comparison

SMC

0.83

0.85

0.83

0.98

0.85

0.49

0.88

$\underline{\underline{\sigma}}$

c

Figure 6 Comparison of data derived from FAF, OCT, MP and pRF mapping for seven patients with RP. The left column displays the FAF results while the second column SD-OCTs for every subject showing peripheral macular areas of outer retinal atrophy corresponding to the functional findings. The third column shows MP data, which were first binarised and then interpolated. The fourth column shows the binarised pRF coverage of each subject. The fifth column shows the SMC of MP versus pRF mapping, which was calculated based on the 41 MP test points and pRF mapping data sampled from the pRF coverage map at these locations. FAF, fundus autofluorescence; MP, microperimetry; pRF, population-receptive field; $\mathrm{RP}_{1}$ retinitis pigmentosa; SD-OCT, spectral domain optical coherence tomography; SMC, simple matching coefficient.

also during therapy, the relatively small sample size still limits the ability to extrapolate to the full patient population.

In conclusion, a novel approach to link conventional testing including MP and structural imaging to retinotopic mapping of the primary visual cortex is presented using fMRI in patients with STGD and RP. pRF coverage maps provide objective functional data independent of conscious perception and feedback by the patient and are a valuable adjunct in the assessment of retinal 
dysfunction. The addition of pRF data to conventional testing in patients with retinal disease could demonstrate other aspects of visual function not reflected in standard clinical measures, for example, cortical reorganisation or unexplained visual sparing. The results of this study reinforce the possibility of fMRI having a clinical application in the future.

Contributors Design and conduct of the study: MR, AH, AAL, GEH, CW, UMS-E; collection and management of the data: MR, AH, AAL; analysis and interpretation of the data: MR, AH, AAL, GEH, CW, UMS-E; preparation of the manuscript: MR, AH; and review of the manuscript: GEH, CW, UMS-E.

Funding This research received no specific grant from any funding agency in the public, commercial or not-for-profit sectors.

Competing interests None declared.

Patient consent Obtained.

Ethics approval The protocol was approved by the local ethics committee (Ethikkommission der Medizinischen Universität Wien) and adhered to the tenets of the Declaration of Helsinki.

Provenance and peer review Not commissioned; externally peer reviewed.

(c) Author(s) (or their employer(s)) 2019. No commercial re-use. See rights and permissions. Published by BMJ.

\section{REFERENCES}

1 Hanout M, Horan N, Do DV, . Introduction to microperimetry and its use in analysis of geographic atrophy in age-related macular degeneration. Curr Opin Ophthalmol 2015;26:149-56.

2 Wu Z, Ayton LN, Guymer RH, et al. Intrasession test-retest variability of microperimetry in age-related macular degeneration. Invest Ophthalmol Vis Sci 2013;54:7378-85.

3 Chen FK, Patel PJ, Xing W, et al. Test-retest variability of microperimetry using the Nidek MP1 in patients with macular disease. Invest Ophthalmol Vis Sci 2009;50:3464-72.

4 Wandell BA, Dumoulin SO, Brewer AA. Visual field maps in human cortex. Neuron 2007:56:366-83.

5 Dumoulin SO, Wandell BA. Population receptive field estimates in human visual cortex. Neuroimage 2008;39:647-60.

6 Wandell BA, Winawer J. Computational neuroimaging and population receptive fields Trends Cogn Sci 2015;19:349-57.

7 Sunness IS, Liu T, Yantis S. Retinotopic mapping of the visual cortex using functional magnetic resonance imaging in a patient with central scotomas from atrophic macular degeneration. Ophthalmology 2004;111:1595-8.
8 Baseler HA, Gouws A, Crossland MD, et al. Objective visual assessment of antiangiogenic treatment for wet age-related macular degeneration. Optom Vis Sci 2011;88:1255-61.

9 Baseler HA, Gouws A, Haak KV, et al. Large-scale remapping of visual cortex is absent in adult humans with macular degeneration. Nat Neurosci 2011;14:649-55.

10 Liu T, Cheung SH, Schuchard RA, et al. Incomplete cortical reorganization in macular degeneration. Invest Ophthalmol Vis Sci 2010;51:6826-34.

11 Masuda Y, Dumoulin SO, Nakadomari S, et al. V1 projection zone signals in human macular degeneration depend on task, not stimulus. Cereb Cortex 2008;18:2483-93.

12 Baker Cl, Dilks DD, Peli E, et al. Reorganization of visual processing in macular degeneration: replication and clues about the role of foveal loss. Vision Res 2008:48:1910-9

13 Dilks DD, Baker Cl, Peli E, et al. Reorganization of visual processing in macular degeneration is not specific to the "preferred retinal locus". J Neurosci 2009:29:2768-73.

14 Schumacher EH, Jacko JA, Primo SA, et al. Reorganization of visual processing is related to eccentric viewing in patients with macular degeneration. Restor Neurol Neurosci 2008;26(4-5):391-402

15 Ferreira S, Pereira AC, Quendera B, et al. Primary visual cortical remapping in patients with inherited peripheral retinal degeneration. Neuroimage Clin 2017;13:428-38.

16 Lois N, Holder GE, Bunce C, et al. Phenotypic subtypes of stargardt macular dystrophyfundus flavimaculatus. Arch Ophthalmol 2001;119:359-69.

17 McCulloch DL, Marmor MF, Brigell MG, et al. ISCEV Standard for full-field clinical electroretinography (2015 update). Doc Ophthalmol 2015;130:1-12.

18 Fujii GY, de Juan E, Sunness J, et al. Patient selection for macular translocation surgery using the scanning laser ophthalmoscope. Ophthalmology 2002;109:1737-44.

19 Moeller S, Yacoub E, Olman CA, et al. Multiband multislice GE-EPI at 7 tesla, with 16-fold acceleration using partial parallel imaging with application to high spatial and temporal whole-brain fMRI. Magn Reson Med 2010;63:1144-53.

20 Power JD, Barnes KA, Snyder AZ, et al. Spurious but systematic correlations in functional connectivity MRI networks arise from subject motion. Neuroimage 2012:59:2142-54.

21 Hummer A, Ritter M, Tik M, et al. Eyetracker-based gaze correction for robust mapping of population receptive fields. Neuroimage 2016;142:211-24.

22 Hummer A, Ritter M, Woletz M, et al. Artificial scotoma estimation based on population receptive field mapping. Neuroimage 2018;169:342-51.

23 Qing G, Zhang S, Wang B, et al. Functional MRI signal changes in primary visual cortex corresponding to the central normal visual field of patients with primary open-angle glaucoma. Invest Ophthalmol Vis Sci 2010;51:4627-34.

24 Papanikolaou A, Keliris GA, Papageorgiou TD, et al. Population receptive field analysis of the primary visual cortex complements perimetry in patients with homonymous visual field defects. Proc Nat/ Acad Sci U S A 2014;111:E1656-5.

25 Goesaert E, Van Baelen M, Spileers W, et al. Visual space and object space in the cerebral cortex of retinal disease patients. PLoS One 2014;9:e88248.

26 Masuda Y, Horiguchi H, Dumoulin SO, et al. Task-dependent V1 responses in human retinitis pigmentosa. Invest Ophthalmol Vis Sci 2010;51:5356-64. 